\title{
Anaesthetic Management of Tracheal Agenesis
}

\author{
Bhattarai B, ${ }^{1}$ Pratap A, ${ }^{2}$ Yadav R, ${ }^{1}$ Pokharel $K^{1}$ \\ 'Department of Anaesthesiology and Critical Care, ${ }^{2}$ Department of Surgery, BP Koirala Institute of Health Sciences (BPKIHS), \\ Dharan, Nepal
}

\section{ABSTRACT}

Tracheal agenesis is a rare but uniformly fatal congenital condition that obstetricians, neonatologists or anaesthesiologists may encounter as an unexpected emergency. Ventilation with bag and mask or through an endotracheal tube placed in the oesophagus may be the only options left for immediate survival and evaluation. Ventilation and maintenance of adequate oxygenation can be difficult during surgical interventions. We report a case of neonate in which ventilation and anaesthesia were maintained through an endotracheal tube in the oesophagus and frequent episodes of drop in oxygen saturation were observed during surgery. Anaesthetic management, airway and other issues are discussed.

Key Words: anaesthetic, airway, management, tracheal agenesis

\section{INTRODUCTION}

Tracheal agenesis (TA) is a rare congenital anomaly associated with uniformly fatal outcomes. This condition manifests at birth with respiratory distress and absence of audible cry. Obstetricians, neonatologists and anaesthesiologist may encounter this condition as an unexpected emergency. Ventilation with bag and mask or through an endotracheal tube in the oesophagus may be the only options left for immediate survival and evaluation. ${ }^{1}$ Reports on the experience about anaesthetic management of TA are limited or lacking. We report this case to share our experience of anaesthetic management of a case of tracheal agenesis with broncho-oesophageal fistula in which neck exploration, oesophagotracheostomy, cervical oesophagostomy, thoracotomy, ligation of lower end of oesophagus and gastrostomy were performed.

\section{CASE REPORT}

A male neonate born to a 25 year healthy mother through lower segment caesarean section (for leaking $\mathrm{P} / \mathrm{V}$ and foetal distress) at 28 weeks of gestation was suspected to have tracheal agenesis (TA) after numerous failed attempts of tracheal intubation despite clear visualization of vocal cords on laryngoscopy and possibility of ventilation with bag and mask as well as through endotracheal tube in the oesophagus. Paediatric surgeons planned neck exploration under general anaesthesia. The neonate was ventilated through an endotracheal tube in the oesophagus using a self-inflating resuscitator with supplemental oxygen. The neonate weighed $2.0 \mathrm{~kg}$ and had the APGAR score of 1 and 4 at 1 and 5 minutes respectively. Clinically, the neonate was cyanosed and had crepitations and conducted sounds in both sides of the chest on

\author{
Correspondence: \\ Dr. Balkrishna Bhattarai \\ Department of Anaesthesiology and Critical Care \\ BPKIHS, Dharan, Nepal. \\ Phone: +977-25-521017 Ext. 2012 \\ Email: bhattaraibk@yahoo.com
}


Bhattarai et al. Tracheal agenesis: anaesthetic management

auscultation. Cardiovascular system evaluation revealed a normal heart rate of 130 beats per minute and normal heart sounds. Preoperative laboratory investigations were within normal limits except the arterial blood gases which showed $\mathrm{pH} 7.24, \mathrm{PO}_{2} 47.4 \mathrm{mmHg}, \mathrm{PCO}_{2}$ $29.5 \mathrm{mmHg}$ and $\mathrm{HCO}_{3} 16.2 \mathrm{mmol} /$ litre. Chest x-ray showed stomach position of the nasogastric tube. Intraoperative monitoring included ECG, pulse oximetry and non-invasive blood pressure (NIBP).

Initial (prior to induction of anaesthesia) $\mathrm{SPO}_{2}$ was $88 \%$, heart rate was 134 beats per minute and blood pressure of $80 / 50 \mathrm{mmHg}$. General anaesthesia was induced with $8 \mathrm{mg}$ of thiopentone sodium and $1 \mathrm{mg}$ of pethidine intravenously. Injection vecuronium 0.2 $\mathrm{mg}$ was used for muscle relaxation. Anaesthesia was maintained with $0.5-1.0 \%$ of halothane in oxygen through the endotracheal tube in the oesophagus.

Neck exploration by the surgeons revealed absence of trachea beyond the level of thyroid cartilage and oesophageal position of the endotracheal tube confirming the diagnosis of TA (Figure 1). Exploration of oesophagus through right thoracotomy showed two main bronchi arising from the oesophagus. Surgeons ligated the oesophagus distal to the fistula to prevent reflux of the stomach content. After ligation of the distal oesophagus, ventilation became more effective with $\mathrm{SPO}_{2}$ increasing to $97 \%$. A $4.0 \mathrm{~mm}$ internal diameter endotracheal tube was inserted for subsequent ventilation into the distal oesophagus after making a double-barrel cervical oesophagostomy by the surgeons. A gastrostomy was made for feeding and gastric aspiration. Total duration of surgery was two and half hours. Estimated total blood loss was 40 $\mathrm{ml}$. Total of $200 \mathrm{ml}$ of crystalloid ( $\mathrm{N} / 5$ saline in $5 \%$ dextrose) was infused during intraoperative period. The patient remained haemodynamically stable but there were frequent drops in $\mathrm{SPO}_{2}$ down to the level of $70 \%$ particularly at the times of manipulation of the oesophagus by the surgeons. The surgeons were informed about the drop in oxygen saturation and were asked to release or relax the traction for the time being.

On completion of surgery, the patient was shifted to the neonatal ICU for mechanical ventilation and further management where the patient developed sepsis and died after 5 days.

\section{DISCUSSION}

Tracheal agenesis is a rare condition of unclear aetiology that an anaesthesiologist may encounter during airway management or resuscitation. ${ }^{2}$ Unlike other common causes of neonatal respiratory distress such as birth asphyxia, prematurity and meconium aspiration, TA does not respond to usual clinical resuscitation measures. The severity of the condition can range from total pulmonary atresia to tracheal stenosis. Classification of TA by Faro and colleagues based on the decreasing severity of the anomalies $A$ (total pulmonary atresia) through G (tracheal stenosis) is useful due to its simplicity and practicality. ${ }^{3}$ With main bronchi arising from the oesophagus separately, our case falls into Faro type B.

Failure to advance tracheal tube despite clearly visible vocal cords and possibility of bag and mask ventilation in our case suggested presence of tracheal agenesis. Oesophageal intubation and ventilation are tried only after more common causes of respiratory distress are excluded. ${ }^{1}$ Intubation while the newborn is awake and breathing spontaneously is advisable. ${ }^{4}$ Presence of tracheo-oesophageal or broncho-oesophageal fistula is essential for survival; its presence is associated with successful ventilation which was true in our case also. ${ }^{5,6}$

We experienced frequent episodes of difficulty in ventilation and drop in $\mathrm{SPO}_{2}$ in the neonate during surgical manipulation of the oesophagus. This is obviously explained by the placement of the tracheal tube in the collapsible oesophagus in which small pull by the surgeon can occlude the lumen. Further, loose fit of the endotracheal tube in the oesophagus and escape of gas can contribute to the ineffectiveness of ventilation. Every episode of drop in oxygen saturation could be managed by continuous communication and cooperation between the anaesthesiologists and the surgeons. However, after the surgeon ligated the distal oesophagus (beyond the fistula) and put the

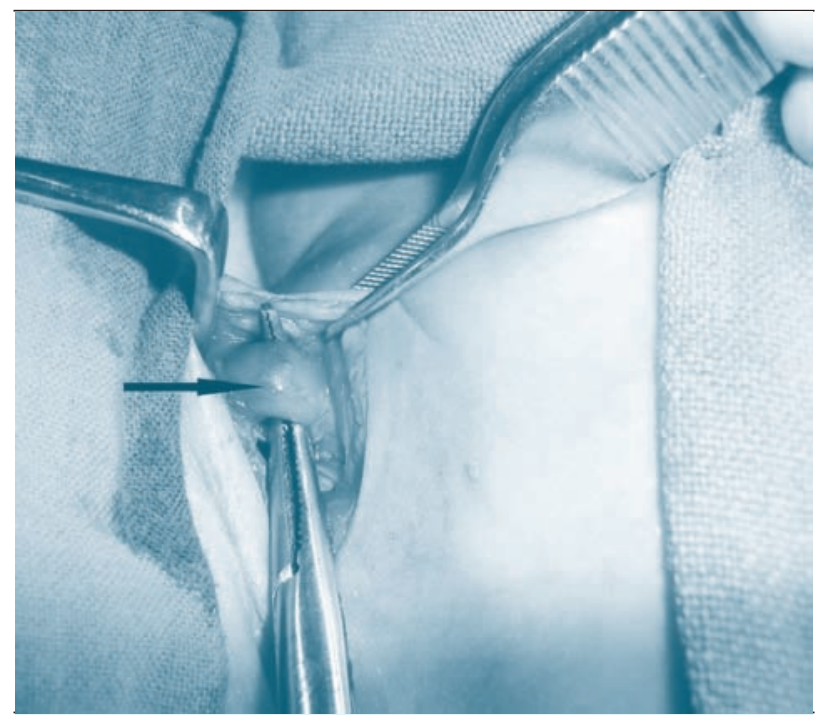

Figure 1: Photograph showing neck exploration. Absence of trachea and the presence of the endotracheal tube in the oesophagus is evident (black arrow). 
endotracheal tube in the distal oesophagus, ventilation became easier with improved oxygenation.

Although not found in our case, there may be other important anaesthetic concerns related to associated anomalies known as VACTERL (vertebral, anal, cardiac, tracheo-oesophageal, renal and limb anomalies). ${ }^{3}$

Despite attempts of various surgical approaches long term survival are yet to be achieved. Baroncini-Cornea
$S$ and colleagues have reported on management of 10 months of life in a case of TA. ${ }^{7}$ But in their case, there was small segment of trachea suggesting it to be of Faro's Type E. The reported survival in case of more severe forms of TA (such as Faro type B in our case) is 1-6 weeks with pneumonia as the usual cause of death. ${ }^{8}$ Unfortunately, attempts of using synthetic or homologous grafts for tracheal reconstruction have not been encouraging. ${ }^{9,10}$

\section{REFERENCES}

1. van Veenendaal MB, Liem KD, Marres HAM. Congenital absence of trachea. Eur J Pediatr 2000; 159: 8-13.

2. De Jose Maria B, Drudis R, Monclus E, Silva A, Santander $\mathrm{S}$, Cusi V. Management of tracheal stenosis. Paediatric Anaesthesia 2000; 10: 441-4.

3. Faro RS, Goodwin CD, Organ CH Jr. Tracheal agenesis. Ann Thorac Surg 1979; 28: 295-9.

4. Saleeby MG, Vustar M, Algren J. Tracheal agenesis: a rare disease with unique airway consideration. Anesth Analg 2003; 97: 50-2.

5. Lyons SM, Bruce AEG. Tracheal agenesis: a report of two cases. Anaesthesia 1968; 23: 98-102.

6. McNie DJM, Pryse-Davies J. Tracheal agenesis. Arch Dis Child 1970; 45: 143-4.
7. Baroncini-Cornea S, Fae M, Gargiulo G, Gentili A, Lima M, Pigna A, et al. Tracheal agenesis: management of the first 10 months of life. Pediatric Anesthesia 2004; 14: 774-7.

8. Kerschner J, Klotch DW. Tracheal agenesis: a case report and review of literature. Otolaryngol Head Neck Surg 1997; 116: 123-8.

9. Neville WE, Bolonowski PJP, Soltanzadek H. Prosthetic reconstruction on trachea and carina. J Thoracic Cardiovasc Surg 1976; 72: 525-38.

10. Jacobs JP, Quintessenza JA, Andrews T, Burke RP, Spektor Z, Delius RE, et al. Tracheal allograft reconstruction: the total North American and worldwide pediatric experiences. Ann Thorac Surg 1999; 68: 1043-51. 